\title{
THE VALIDITY OF BEURLING THEOREMS IN POLYDISCS
}

\author{
V. MANDREKAR
}

(Communicated by Paul S. Muhly)

\begin{abstract}
This paper gives necessary and sufficient conditions for an invariant subspace $\mathscr{M}$ of $H^{2}\left(T^{2}\right)$ to be of the form $q H^{2}\left(T^{2}\right)$ ( $q$ inner) in terms of double commutativity of the shifts. Recent results in [8] follow directly from our work. Relation to the work in $[\mathbf{1}]$ is also discussed.
\end{abstract}

In [5], Rudin gave an example of a shift-invariant subspace of $H^{2}\left(T^{2}\right)$ which is not of the form $q H^{2}\left(T^{2}\right)$, where $q$ is an inner function. In addition, [5] gives an example of an "outer function" $f$ for which $\mathscr{M}_{f}$, the smallest invariant subspace generated by $f$ is not equal to $H^{2}\left(T^{2}\right)$. Motivated from the prediction theory for random fields, Soltani [8] gave complicated necessary and sufficient conditions for an "outer function" in the sense of [5] to satisfy $\mathscr{M}_{f}=H^{2}\left(T^{2}\right)$. Our purpose here is to use the Theorem of Halmos for two commuting isometries due to Slocinski [7] to obtain necessary and sufficient conditions on the invariant subspace $\mathscr{M}$ of $H^{2}\left(T^{2}\right)$ to be of the form $q H^{2}\left(T^{2}\right)$ ( $q$ inner). As a consequence of this result we obtain necessary and sufficient conditions on $\mathscr{M}_{f}$ for an outer function $f$, to be $H^{2}\left(T^{2}\right)$. We use results of [3] to relate our conditions to those in [8]. As a by-product of our main theorem we show that all invariant subspaces unitarily equivalent to $q H^{2}\left(T^{2}\right)$ are exactly of the same form. The last result relates to some recent work of Agrawal, Clark, and Douglas [1], where the problem of unitary equivalence of invariant subspaces of $H^{2}\left(T^{n}\right)$ was solved under some sufficient conditions. Their case does not include subspaces of the type considered here. We thank the referee and the editor for bringing the work in [1] to our attention. We begin with some notation.

Let $\mathbf{Z}$ be the set of integers. We denote by $m, n$ etc. the elements of $\mathbf{Z}$. Let $U$ be the open unit disc and $T$ the boundary of $U$ in the complex plane $\mathbf{C}$. Let $\mathbf{Z}^{2}, \mathbf{C}^{2}, U^{2}$ and $T^{2}$ be the respective cartesian products and $\sigma_{2}$ the normalized Lebesgue measure on $T^{2}$. For $p>0$, we denote by $L^{p}\left(T^{2}, \sigma_{2}\right)$ the usual Lebesgue space of the equivalence class of $p$-integrable functions and

$$
H^{p}\left(U^{2}\right)=\left\{f: f: U^{2} \rightarrow \mathbf{C} \text { analytic and } \sup _{0 \leq r \leq 1} \int_{T}\left|f_{r}(\mathbf{t})\right|^{p} d \sigma_{2}<\infty\right\} .
$$

Here $f_{r}(\mathbf{t})=f(z)$ with $z=r \mathbf{t}$. Let $\mathbf{z}=\left(z_{1}, z_{2}\right)=\left(r_{1} e^{i \theta_{1}}, r_{2} e^{i \theta_{2}}\right)$ and $\mathbf{t}=$ $\left(e^{i \theta_{1}}, e^{i \theta_{2}}\right)$, then $P(\mathbf{z}, \mathbf{t})=P_{r_{1}}\left(\theta_{1}-\theta_{2}\right) . P_{r_{2}}\left(\theta_{1}-\theta_{2}\right)$ is called Poisson kernel with

Received by the editors March 30, 1987.

1980 Mathematics Subject Classification (1985 Revision). Primary 32A30, 60G10.

Key words and phrases. Polydisc, Beurling Theorem.

Supported in part by ONR N00014-85-K-0150 and the Air Force Office of Scientific Research Contract No. F49620 85 C 0144. 
$P_{r}(\theta)=\left(1-r^{2}\right) /\left(1-2 r \cos \theta+r^{2}\right)$. It is known that for $f \in H^{p}\left(U^{2}\right), \lim _{r \rightarrow 1} f_{r}(\mathbf{t})=$ $f^{*}(\mathbf{t})$ exists and is in $L^{p}\left(T^{2}, \sigma_{2}\right)$. For $f \in L^{p}\left(T^{2}, \sigma_{2}\right)$, let $f^{e}(z)=\int_{T^{2}} P(\mathbf{z}, \mathbf{t}) f(t) d \sigma_{2}$, then $f^{e} \in H^{p}\left(U^{2}\right)$. In case $p=2, f^{e} \in H^{2}\left(U^{2}\right)$ if $f \in L^{2}\left(T^{2}, \sigma_{2}\right)$ and $f(\mathbf{t})=$ $\sum_{m=0} \sum_{n=0} a_{m n} t_{1}^{m} t_{2}^{n}$ and $f^{e}=\sum_{m=0} \sum_{n=0} a_{m n} z_{1}^{m} z_{2}^{n}$. Conversely, every $f \in$ $H^{2}\left(U^{2}\right)$ has this form and $f^{*}(\mathbf{t})=\sum_{m=0} \sum_{n=0} a_{m n} t_{1}^{m} t_{2}^{n}$. For further information see $[5]$.

In order to characterize invariant subspaces of the form $q H^{2}$ in terms of the section of the shifts on it, we note that subspaces of the form $q H^{2}$ can be represented as

$$
q H^{2}=\sum_{m=0}^{\infty} \sum_{n=0}^{\infty} \bigoplus V_{1}^{m} V_{2}^{n}(M)
$$

where $M$ equals the span of $q$ in $H^{2}\left(T^{2}\right)$ and $V_{i}$ is the multiplication by $t_{i}$ on $H^{2}\left(T^{2}\right)$ with $\mathbf{t}=\left(t_{1}, t_{2}\right) \in T^{2}$. As $V_{1}$ commutes with $V_{2}$ (in short, $V_{1} \sim V_{2}$ ), we get from (1) and Theorem 1 (i) $\Rightarrow$ (ii) of [7] that $V_{1}$ and $V_{2}$ are doubly commuting (i.e. $\left.V_{1} \sim V_{2}, V_{1} \sim V_{2}^{*}\right)$. In fact, we have

2. THEOREM. An invariant subspace $\mathscr{M} \neq\{0\}$ of $H^{2}\left(T^{2}\right)$ is of the form $q \cdot H^{2}$ with $q$ inner function if and only if $V_{1}$ and $V_{2}$ are doubly commuting on $\mathscr{M}$.

ProOF. Necessity was proved above. To prove the sufficiency we get, in view of Theorem 1 ((ii) $\Rightarrow($ iv $))[7]$,

$$
\mathscr{M}=\sum_{m=0}^{\infty} \sum_{n=0}^{\infty} \bigoplus V_{1}^{m} V_{2}^{n}\left(R_{1}^{\perp} \cap R_{2}^{\perp}\right),
$$

where $R_{i}^{\perp}=\mathscr{M} \ominus V_{i} \mathscr{M}$. From Theorem 1 (i) $\Leftrightarrow(\mathrm{iv})$ and Corollary 1 of $[\mathbf{7}, \mathrm{p}$. 256] we get that $R_{1}^{\perp} \cap R_{2}^{\perp}=\{0\}$ implies $\mathscr{M}=\{0\}$ giving contradiction. Hence $R_{1}^{\perp} \cap R_{2}^{\perp} \neq\{0\}$. We shall now prove that $R_{1}^{\perp} \cap R_{2}^{\perp}$ is one-dimensional. The above argument shows that dimension of $R_{1}^{\perp} \cap R_{2}^{\perp}$ is at least one. It remains to prove that it is not greater than one. Let $q_{1}, q_{2} \in R_{1}^{\perp} \cap R_{2}^{\perp}$, then $\int_{T^{2}} t_{1}^{m} t_{2}^{n} q_{1} q_{2} d \sigma_{2}=0$ for $m, n>0$. As $V_{2}\left(R_{1}^{\perp}\right) \subseteq\left(R_{1}^{\perp}\right)$ [7, Theorem 1 (iii)], we get $\int_{T^{2}} t_{2}^{n} q_{1} \overline{t_{1}^{m} q_{2}} d \sigma_{2}=0$ for all $n>0$ and $m>0$. Since $t_{1}^{m}=t_{1}^{-m}$, by the symmetry of the problem, we get for $(m, n) \neq(0,0) \int t_{1}^{m} t_{2}^{n} q_{1} \bar{q}_{2} d \sigma_{2}=0$. Since $q_{1} \bar{q}_{2} \in L^{1}\left(T^{2}, \sigma_{2}\right)$ we get $q_{1} \bar{q}_{2}=c$ a.e. $\sigma_{2}$. Suppose now that $q_{1}, q_{2} \in R_{1}^{\perp} \cap R_{2}^{\perp} q_{1} \neq 0, q_{2} \neq 0$ and $q_{1} \perp q_{2}$. This implies that $\left|q_{1}\right|^{2}=c_{1} \neq 0$ a.e. $\sigma_{2},\left|q_{2}\right|^{2}=c_{2} \neq 0$ a.e. $\sigma_{2}$ but $q_{1} \bar{q}_{2}=0$. The last statement follows from the fact $q_{1} \perp q_{2}$ and a.e. $\sigma_{2}, q_{1} \bar{q}_{2}=c$ giving $c=0$ (integrating with respect to $\sigma_{2}$ ). This is impossible. Hence $R_{1}^{\perp} \cap R_{2}^{\perp}$ is one dimensional. Also $q$ generating $R_{1}^{\perp} \cap R_{2}^{\perp}$ is an inner function. Assume $|q|=1$ a.e. choosing $q$ of norm 1. Now (3) gives the result.

In a recent paper [1], Agrawal, Clark and Douglas study the question of unitary equivalence of invariant subspaces of $H^{2}$ of polydiscs. Under the assumption of full range of an invariant subspace $\mathscr{M}$ (for definition see [1, (2), p. 5]), they show that all invariant subspaces $\mathscr{N}$ of $H^{2}$ unitarily equivalent to $\mathscr{M}$ are of the form $\varphi \mathscr{M}$ ( $\varphi$ an inner function). Unfortunately, subspaces of the form $q H^{2}$ do not have full range [1, Remark 3, p. 6] unless $q$ is constant. We use our result to study all invariant subspaces unitarily equivalent to $q H^{2}$. We consider the case of subspaces of $H^{2}\left(T^{2}\right)$. 
If $\mathscr{M}$ is an invariant subspace unitarily equivalent to $q H^{2}$ for some inner function, then in view of Theorem 2 , we get $V_{1}$ and $V_{2}$ are doubly commuting on $\mathscr{M}$. Hence $\mathscr{M}=q^{\prime} H^{2}$ for some inner function $q^{\prime}$ by Theorem 2 with $\left|q^{\prime}(\mathbf{t})\right|=1$ a.e. $\sigma_{2}$. We thus have the following.

4. THEOREM. (a) The class of all invariant subspaces of the form $q H^{2}, q$ inner function with $|q(\mathbf{t})|=1$ a.e. $\sigma_{2}$ are unitarily equivalent.

(b) Any invariant subspace $\mathscr{M}$ of $H^{2}\left(T^{2}\right)$ unitarily equivalent to $\mathscr{N}=q H^{2}$ of the above form is of the same form.

REMARK. Note that in (b) $\mathscr{M}=q^{\prime} / q \mathscr{N}$. Comparing this with Lemma 1 of [1] we get that the function $\varphi=q^{\prime} / q$ with $q^{\prime}$ and $q$ relatively prime in the sense of $[\mathbf{1}$, p. 4].

In [6], Rudin gives an example of the subspace $\mathscr{M}=q^{\prime} / q \mathscr{N}$ with $q^{\prime}, q$ inner but not relatively prime. We should also note that there exists subspaces of the form $q H^{2}$ which do not satisfy analytic condition used in $\S 3$ of $[\mathbf{1}]$.

The example of Rudin [5] mentioned in the introduction shows that there exists $f \in H^{2}$ such that

$$
\mathscr{M}_{f}=\overline{s p}\left\{V_{1}^{m} V_{2}^{n} f ; m, n \geq 0\right\}
$$

is not of the form $q H^{2}$. The following Corollary gives conditions on $\mathscr{M}_{f}$ for this to hold.

5. COROLLARY. $\mathscr{M}_{f}=q H^{2}\left(T^{2}\right)$ if and only if $V_{1}$ and $V_{2}$ are doubly commuting on $\mathscr{M}_{f}$.

Following Helson [2], we say that a function $g$ is $H$-outer if $\mathscr{M}_{g}=H^{2}\left(T^{2}\right)$.

6. COROLlaRY. A function $f \in H^{2}\left(T^{2}\right)$ has the property $f=q \cdot g$ with $q$ inner and $g H$-outer if and only if $V_{1}$ and $V_{2}$ doubly commute on $\mathscr{M}_{f}$.

ProOF. By Corollary 5, only if part follows as $\mathscr{M}_{f}=q H^{2}\left(T^{2}\right)$. To prove the converse, we note that by Corollary $5, \mathscr{M}_{f}=q H^{2}\left(T^{2}\right)$ given $f=q \cdot g, g \in H^{2}\left(T^{2}\right)$. Hence $\mathscr{M}_{f}=q \cdot \mathscr{M}_{g}$ giving $g$ is $H$-outer.

In [5, p. 73], a function $f \in H^{2}\left(U^{2}\right)$ is called outer (we call it $R$-outer) if $\log |f(z)|=\int_{T^{2}} P(z, t) \log \left|f^{*}\right| d \sigma_{2}$. Given a function $f \in H^{2}\left(T^{2}\right)$, we denote by $f^{e} \in H^{2}\left(U^{2}\right)$ the function given by $\int_{T^{2}} P(z, t) f(\mathbf{t}) d \sigma_{2}$. In this case we note that $\left(f^{e}\right)^{*}=f$. It is already known [5, Theorem 4.4.6] that $f$ is $H$-outer then $f^{e}$ is $R$-outer. From this we get in view of Corollary 5 the following.

7. COROLlaRY. Let $g$ be $H$-outer then $g^{e}$ is $R$-outer and $V_{1}$ and $V_{2}$ doubly commute on $\mathscr{M}_{g}$.

We now prove the converse of Corollary 7. Assume now that $V_{1}, V_{2}$ doubly commute on $\mathscr{M}_{f}$ and $f^{e}$ is $R$-outer then by Corollaries 6 and 7 , the definition of $f^{e}, g^{e}$ and the fact that $|q|=1$ we get $f^{e}=p g^{e}$ with $|p|=1$. Thus we get that the slice function $f_{w}^{e}(\lambda)=p_{w}(\lambda) g_{w}^{e}(\lambda)$. Using Lemma 4.4.4(a) of [5] and the uniqueness of outer function [2, p. 13] we get $p_{w}(\lambda)=1$ for all $w$ and $\lambda$ giving $p=1$ i.e., $f=g$. 
Combining this with Corollary 7 gives

8. CoRollary. Let $f \in H^{2}\left(T^{2}\right)$ then $\mathscr{M}_{f}=H^{2}\left(T^{2}\right)$ if and only if $f^{e}$ is $R$-outer and $V_{1}$ and $V_{2}$ doubly commute.

In view of Theorem 4.2 of [3], we get that Corollary 7 includes Beurling Theorem proved in [8, Theorem 1.5]. Now using essentially classical techniques $[4,3]$ one can derive associated results in prediction theory given in [8].

\section{REFERENCES}

1. O. P. Agrawal, D. N. Clark and R. G. Douglas, Invariant subspaces in the polydisk, Pacific J. Math. 121 (1986), 1-11.

2. H. Helson, Lectures on invariant subspaces, Academic Press, New York, 1964.

3. G. Kallianpur and V. Mandrekar, Non-deterministic random fields and Wold and Halmos decompositions for commuting isometries, Prediction Theory and Harmonic Analysis (V. Mandrekar and H. Salehi, eds.), North-Holland, Amsterdam, 1983.

4. P. Masani, Shift-invariant spaces and prediction theory, Acta Math. 107 (1962), 275-290.

5. W. Rudin, Function theory in polydiscs, Benjamin, New York, 1979.

6. Invariant subspaces of $H^{2}$ on a torus, J. Functional Analysis 61 (1985), 378-384.

7. M. Slocinski, On Wold-type decomposition of a pair of commuting isometries, Ann. Polon. Math. 37 (1980), 255-262.

8. A. R. Soltani, Extrapolation and moving average representation for stationary random fields and Beurling's Theorem, Ann. Probab. 12 (1984), 120-132.

Department of Mathematics, Michigan State University, East lansing, MICHIGAN 48824 